\title{
Ranking-Based Semantics for Sets of Attacking Arguments
}

\author{
Bruno Yun, ${ }^{1}$ Srdjan Vesic, ${ }^{2}$ Madalina Croitoru ${ }^{3}$ \\ ${ }^{1}$ University of Aberdeen, Scotland \\ ${ }^{2}$ CRIL - CNRS, Univ. Artois, France \\ ${ }^{3}$ University of Montpellier, France \\ \{yun, croitoru\}@lirmm.fr; vesic@ cril.fr;
}

\begin{abstract}
Argumentation is a process of evaluating and comparing sets of arguments. Ranking-based semantics received a lot of attention recently. All of the semantics introduced so far are applicable to binary attack relations. In this paper, we study a more general case when sets of arguments can jointly attack an argument. We generalise existing postulates for rankingbased semantics to fit this framework, define a general variant of h-categoriser, prove that it converges for every argumentation framework and study the postulates it satisfies. We also study the link between binary and hypergraph version of hcategoriser.
\end{abstract}

\section{Introduction}

Argumentation is the process of reasoning in presence of inconsistent information by exchanging, evaluating or ranking interactive arguments. The most popular way of representing this argumentation process was proposed by Dung in his seminal paper (Dung 1995) which sees arguments as nodes and attacks as edges in a directed graph. Then, one can apply the several argumentation semantics (Baroni, Caminada, and Giacomin 2011) to extract meaningful subsets of arguments called extensions. However, for many applications, this two level of acceptance (arguments belong or not to an extension) was not enough (Leite and Martins 2011). Against this background, a solution consisted in having many levels of acceptability by ranking arguments using the several ranking-based semantics defined in the literature (Amgoud and Ben-Naim 2013; Cayrol and Lagasquie-Schiex 2005; Leite and Martins 2011; Matt and Toni 2008) for the Dung's framework along with a set of desirable properties that they should satisfy (Bonzon et al. 2016; Amgoud and Ben-Naim 2013).

Classic Dung argumentation graphs only take into account a binary attack relation. Such binary attack relation implicitly assumes that the logical language used underneath the argument structure is closed under the "and" logical connective. To free oneself from such assumption, Nielsen and Parsons proposed a generalisation of the Dung framework with sets of attacking arguments (Nielsen and Par-

Copyright (c) 2020, Association for the Advancement of Artificial Intelligence (www.aaai.org). All rights reserved. sons 2007). In their framework arguments can jointly attack a single other argument. For instance, let us consider the deductive argumentation framework (Besnard and Hunter 2001), also known as the (support, conclusion) representation, where support is a set of formulas and conclusion is a formula that follows from the support. Now, let us further consider the standard undercut attack relation where an argument attacks another based on the contradiction between the conclusion of the former and a formula in the support of the latter. However, if we are given a less expressive logical language without conjunction, we are unable to model attacks corresponding to ternary conflicts. Let $x, y$ and $z$ be three formulas of the logic that form a ternary conflict. In order to attack the argument $(\{z\}, z)$, one would need to construct the argument $(\{x, y\}, x \wedge y)$ which is impossible if the language does not include conjunction. To this end (Verheij 1996; Nielsen and Parsons 2007) propose to have sets of attacking arguments (i.e. $(\{x\}, x)$ and $(\{y\}, y)$ jointly attack $(\{z\}, z)$ ). Furthermore, (Nielsen and Parsons 2007) extended the classical Dung semantics (stable, preferred, complete and grounded) to this new hypergraph framework. In view of this, we generalise ranking-based semantics for the hypergraph argumentation framework.

The structure of the paper is as follows. In Section 2, we recall the necessary definitions of a hypergraph argumentation framework and ranking-based semantics. In Section 3 , we define some desirable properties that such rankingbased semantics should satisfy. In Section 4, we propose the first ranking-based semantics for hypergraphs argumentation frameworks following the insights from (Besnard and Hunter 2001), we show that this new ranking-based semantics converges and that it is a proper generalisation of the categoriser semantics defined in (Besnard and Hunter 2001) for classic Dung graphs.

\section{Background}

In the next definition, we extend Dung's abstract framework with sets of attacking arguments, i.e. sets of arguments can now jointly attack an argument.

Definition 1 (Hypergraph argumentation framework). A hypergraph argumentation framework is a pair $\mathcal{A S}=(\mathcal{A}, \mathcal{R})$ with $\mathcal{A}$ a finite and non-empty set of arguments and $\mathcal{R} \subseteq$ 


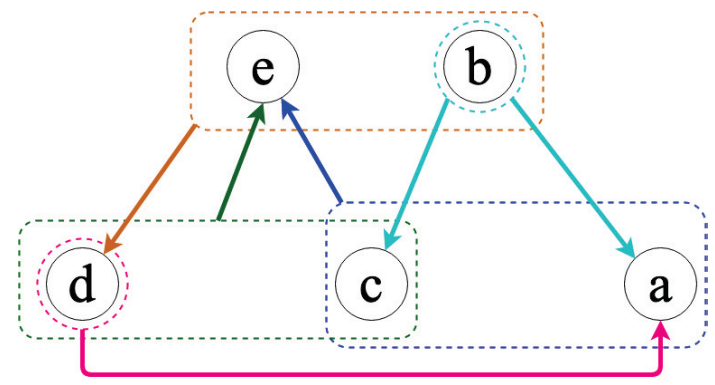

Figure 1: A hypergraph argumentation framework

\section{$\left(2^{\mathcal{A}} \backslash\{\emptyset\}\right) \times \mathcal{A}$.}

Notation. We denote by AS the set of all possible hypergraph argumentation frameworks. Let $\mathcal{A S}=(\mathcal{A}, \mathcal{R}), \mathcal{A S}^{\prime}=$ $\left(\mathcal{A}^{\prime}, \mathcal{R}^{\prime}\right) \in \mathbf{A S}$, we define $\mathcal{A S} \oplus \mathcal{A} \mathcal{S}^{\prime}$ as the argumentation framework $\left(\mathcal{A} \cup \mathcal{A}^{\prime}, \mathcal{R} \cup \mathcal{R}^{\prime}\right)$.

Example 1. In this paper, we will consider the hypergraph argumentation framework $\mathcal{A S}=(\mathcal{A}, \mathcal{R})$ with $\mathcal{A}=\{a, b, c, d, e\}$ and $\mathcal{R}=\{(\{d\}, a),(\{b\}, a),(\{b\}, c)$, $(\{b, e\}, d),(\{d, c\}, e),(\{a, c\}, e)\}$ (see Figure 1).

A ranking-based semantics is a function that returns a ranking on arguments for every hypergraph argumentation framework.

Definition 2 (Ranking-based semantics). A ranking-based semantics $\sigma$ associates to any hypergraph argumentation framework $\mathcal{A S}=(\mathcal{A}, \mathcal{R})$ a ranking $\succeq_{\mathcal{A S}}^{\sigma}$ on $\mathcal{A}$ where $\succeq_{\mathcal{A S}}^{\sigma}$ is a total preorder (reflexive and transitive relation) on $\mathcal{A}$. The notation $a \succeq_{\mathcal{A S}}^{\sigma} b$ means that $a$ is at least as acceptable as $b$.

Notation. As usual, the notation $a \succ_{\mathcal{A S S}}^{\sigma} b$ is used for $a \succeq_{\mathcal{A S}}^{\sigma}$ $b$ and $b \succeq_{\mathcal{A S}}^{\sigma} a$. Likewise, we use $a \simeq_{\mathcal{A S}}^{\sigma} b$ for $a \succeq_{\mathcal{A S}}^{\sigma} b$ and $b \succeq_{\mathcal{A S}}^{\sigma} a$. By abuse of notation, if $S$ and $S^{\prime}$ are two sets of arguments, we write that $S \succeq_{\mathcal{A S S}}^{\sigma} S^{\prime}$ iff for every $s \in S$ and every $s^{\prime} \in S^{\prime}$, we have $s \succeq_{\mathcal{A S}}^{\sigma} s^{\prime}$. Let $S$ be a set in $\left(2^{\mathcal{A}} \backslash\{\emptyset\}\right)$ and $\sigma$ be a ranking-based semantics, the set $\min _{\mathcal{A S}}^{\sigma}(S)$ is the set $\left\{s \in S \mid\right.$ for every $\left.s^{\prime} \in S, s^{\prime} \succeq_{\mathcal{A S}}^{\sigma} s\right\}$.

Definition 3 (Path). Let $\mathcal{A S}=(\mathcal{A}, \mathcal{R}) \in \mathbf{A S}$ and $a \in \mathcal{A}$. We say that a sequence of attacks $\left(\left(S_{1}, t_{1}\right), \ldots,\left(S_{n}, t_{n}\right)\right)$ is a path of size $n$ from $S_{1}$ to $a$ iff for every $i \in$ $\{1, \ldots, n\},\left(S_{i}, t_{i}\right) \in \mathcal{R}, t_{n}=a$ and for every $i \in$ $\{1, \ldots, n-1\}$, it holds that $t_{i} \in S_{i+1}$.

Notation. Let $\mathcal{A S}=(\mathcal{A}, \mathcal{R}) \in \mathbf{A S}, S \in 2^{\mathcal{A}}$ and $a \in \mathcal{A}$, we say that $S \in \mathcal{R}_{n}^{-}(a)$ iff there exists a path of size $n$ from $S$ to $a$. We say that $S \in \mathcal{R}_{1}^{-}(a)$ is a direct attacker of $a$ and $S \in \mathcal{R}_{2}^{-}(a)$ is a direct defender of $a$.

Example 2 (Example 1 Cont'd). The sequence $((\{d\}, a),(\{a, c\}, e))$ is a path of size 2 from $\{d\}$ to $e$. Thus, $\{d\} \in \mathcal{R}_{2}^{-}(e)$ and $\{d\}$ is a direct defender of $e$.

\section{Properties}

We first introduce the definition of an isomorphism between two hypergraph argumentation frameworks.
Definition 4 (Isomorphism). An isomorphism between two hypergraph argumentation frameworks $\mathcal{A S}=(\mathcal{A}, \mathcal{R})$ and $\mathcal{A} \mathcal{S}^{\prime}=\left(\mathcal{A}^{\prime}, \mathcal{R}^{\prime}\right)$ is a bijective function $\gamma: \mathcal{A} \rightarrow \mathcal{A}^{\prime}$ such that for every $S \in 2^{\mathcal{A}}$ and $a \in \mathcal{A},(S, a) \in \mathcal{R}$ iff $(\{\gamma(s) \mid$ $s \in S\}, \gamma(a)) \in \mathcal{R}^{\prime}$. With a slight abuse of notation, we will note $\mathcal{A} \mathcal{S}^{\prime}=\gamma(\mathcal{A S})$.

In the rest of this section, we recall the properties for a ranking-based semantics $\sigma$ that have been defined in the literature (Amgoud and Ben-Naim 2013; Bonzon et al. 2016) and we translate them for hypergraph argumentation frameworks. We now begin with the properties that can be translated straightforwardly.

The Abstraction property states that the name of the arguments should not be taken into account for the ranking.

Property 1 (Abstraction). We say that $\sigma$ satisfies Abstraction iff for any $\mathcal{A S}, \mathcal{A} \mathcal{S}^{\prime} \in \mathbf{A S}$ and isomorphism $\gamma$ such that $\mathcal{A S}^{\prime}=\gamma(\mathcal{A S})$, we have $a \succeq_{\mathcal{A S}}^{\sigma} b$ iff $\gamma(a) \succeq_{\mathcal{A S}^{\prime}}^{\sigma} \gamma(b)$

The Independence property states that two arguments with no paths connecting them should not influence each other.

Property 2 (Independence). We say that $\sigma$ satisfies Independence iff for any $\mathcal{A S}=(\mathcal{A}, \mathcal{R}), \mathcal{A} \mathcal{S}^{\prime}=\left(\mathcal{A}^{\prime}, \mathcal{R}^{\prime}\right)$ in $\mathbf{A S}$ such that $\mathcal{A} \cap \mathcal{A}^{\prime}=\emptyset$ and every $a, b \in \mathcal{A}$ we have $a \succeq_{\mathcal{A S}}^{\sigma} b$ iff $a \succeq_{\mathcal{A S} \oplus \mathcal{A} \mathcal{S}^{\prime}}^{\sigma} b$.

The Void precedence property states that non-attacked arguments should be ranked higher than attacked arguments.

Property 3 (Void precedence). We say that $\sigma$ satisfies Void precedence iff for any $\mathcal{A S}=(\mathcal{A}, \mathcal{R}) \in \mathbf{A} \mathbf{S}$ and $a, b \in \mathcal{A}$ such that $\mathcal{R}_{1}^{-}(a)=\emptyset$ and $\mathcal{R}_{1}^{-}(b) \neq \emptyset$ we have $a \succ_{\mathcal{A S}}^{\sigma} b$.

The Self-contradiction property states that selfcontradicting arguments should be ranked lower than non self-contradicting arguments.

Property 4 (Self-contradiction). We say that $\sigma$ satisfies Selfcontradiction iff for any $\mathcal{A S}=(\mathcal{A}, \mathcal{R}) \in \mathbf{A} \mathbf{S}$ and $a, b \in \mathcal{A}$ such that there exists $S_{1} \in \mathcal{R}_{1}^{-}(a)$ with $a \in S_{1}$ and there exists no $S_{2} \in \mathcal{R}_{1}^{-}(b)$ with $b \in S_{2}$ we have $b \succ_{\mathcal{A S}}^{\sigma} a$.

The Cardinality precedence property states that if an argument $a$ has more attackers than an argument $b$ than it should be ranked lower than $b$. The intuition is that an argument attacked by more sets of arguments is seen as more conflicted (and thus weaker).

Property 5 (Cardinality precedence). We say that $\sigma$ satisfies Cardinality precedence iff for any $\mathcal{A S}=(\mathcal{A}, \mathcal{R}) \in \mathbf{A S}$ and $a, b \in \mathcal{A}$ such that $\left|\mathcal{R}_{1}^{-}(a)\right|>\left|\mathcal{R}_{1}^{-}(b)\right|$ we have $b \succ_{\mathcal{A S}}^{\sigma} a$.

The Defense precedence property states that if $a, b$ are two arguments with the same number of attackers and $a$ is defended but $b$ is not then $a$ should be ranked higher than $b$.

Property 6 (Defense precedence). We say that $\sigma$ satisfies Defense precedence iff for any $\mathcal{A S}=(\mathcal{A}, \mathcal{R}) \in \mathbf{A S}$ and $a, b \in \mathcal{A}$ such that $\left|\mathcal{R}_{1}^{-}(a)\right|=\left|\mathcal{R}_{1}^{-}(b)\right|, \mathcal{R}_{2}^{-}(a)=\emptyset$ and $\mathcal{R}_{2}^{-}(b) \neq \emptyset$ we have $b \succ_{\mathcal{A S}}^{\sigma} a$.

The Total property states that two arguments should always be comparable. 
Property 7 (Total). We say that $\sigma$ satisfies Total iff for any $\mathcal{A S}=(\mathcal{A}, \mathcal{R}) \in \mathbf{A S}$ and $a, b \in \mathcal{A}$ we have $a \succeq_{\mathcal{A S}}^{\sigma} b$ or $b \succ_{\mathcal{A S}}^{\sigma} a$.

The Non-attacked equivalence property states that two non-attacked arguments should be ranked equivalently.

Property 8 (Non-attacked equivalence). We say that $\sigma$ satisfies Non-attacked equivalence iff for any $\mathcal{A S}=(\mathcal{A}, \mathcal{R}) \in$ AS and $a, b \in \mathcal{A}$ such that $\mathcal{R}_{1}^{-}(a)=\mathcal{R}_{1}^{-}(b)=\emptyset$ we have $a \simeq_{\mathcal{A S}}^{\sigma} b$

Definition 5 (Simple and distributed defense). Let $\mathcal{A S}=$ $(\mathcal{A}, \mathcal{R}) \in \mathbf{A S}$ and $a \in \mathcal{A}$. The defense of $a$ is simple iff for every direct defender $S$ of $a$, there is a unique $S^{\prime} \in \mathcal{R}_{1}^{-}(a)$ and $s^{\prime} \in S^{\prime}$ such that $\left(S, s^{\prime}\right) \in \mathcal{R}$. The defense of $a$ is distributed iff for every direct attacker $S$ of $a$, there is at most one direct defender $S^{\prime}$ of $a$ such that $\left(S^{\prime}, s\right) \in \mathcal{R}$ where $s \in S$.

The Distributed-defense precedence property states that if argument $a$ has a simple and distributed defense and argument $b$ has a simple but not distributed defense than $a$ should be higher ranked than $b$.

Property 9 (Distributed-defense precedence). $\sigma$ satisfies Distributed-defense precedence iff for any $\mathcal{A S}=(\mathcal{A}, \mathcal{R}) \in$ $\mathbf{A S}$, for any $a, b \in \mathcal{A}$ such that $\left|\mathcal{R}_{1}^{-}(a)\right|=\left|\mathcal{R}_{1}^{-}(b)\right|$ and $\left|\mathcal{R}_{2}^{-}(a)\right|=\left|\mathcal{R}_{2}^{-}(b)\right|$ and that the defense of $a$ is simple and distributed and that the defense of $b$ is simple but not distributed we have $a \succ_{\mathcal{A S}}^{\sigma} b$.

Definition 6 (Cycle). Let $\mathcal{A S}=(\mathcal{A}, \mathcal{R})$ be a hypergraph argumentation framework, $S \in\left(2^{\mathcal{A}} \backslash\{\emptyset\}\right)$ and $s \in \mathcal{A}$. We say that a path from $S$ to $s$ is a cycle iff $s \in S$. An argumentation framework is called acyclic iff there is no cycle.

An attack (resp. defense) branch of an argument $a$ is a hypergraph argumentation framework such that its fusion with the original argumentation framework should result in the addition of an even (resp. odd) path to $a$.

Definition 7 (Added attack/defense branch). Let $\mathcal{A S}=$ $(\mathcal{A}, \mathcal{R})$ be a hypergraph argumentation framework and $a \in$ A. $P_{+}(a)$ (resp. $\left.P_{-}(a)\right)$ is a pair $\left(\mathcal{A}_{0}, \mathcal{R}_{0}\right)$ called a defense (resp. attack) branch added to $a$ iff:

- $\mathcal{A}_{0}=\left\{a, x_{1}, \ldots, x_{k}\right\}$ and $\mathcal{A} \cap \mathcal{A}_{0}=\{a\}$

- there exists $n \in 2 \mathbb{N}$ (resp. $n \in 2 \mathbb{N}+1), S_{1} \in\left(2^{\mathcal{A}_{0}} \backslash\{\emptyset\}\right)$ and an acyclic path $\left(\left(S_{1}, t_{1}\right), \ldots,\left(S_{n}, t_{n}\right)\right)$ of size $n$ such that $t_{n}=a$.

- $\mathcal{R}_{0}=\left\{\left(S_{i}, t_{i}\right) \mid i \in\{1, \ldots, n\}\right\}$ and $\left(\bigcup_{1}^{n} S_{i}\right) \cup\{a\}=\mathcal{A}_{0}$

The Strict addition of defense branch property states that adding a defense branch to an argument should increase its rank.

Property 10 (Strict addition of defense branch). We say that $\sigma$ satisfies Strict addition of defense branch iff for any $\mathcal{A S}^{\prime}, \mathcal{A S}=(\mathcal{A}, \mathcal{R}) \in \mathbf{A S}$ and any $a \in \mathcal{A}$ such that there exists an isomorphism $\gamma$ with $\mathcal{A S}^{\prime}=\gamma(\mathcal{A S})$ we have $\gamma(a) \succ_{\mathcal{A S} \oplus \mathcal{A S} \mathcal{S}^{\prime} \oplus P_{+}(\gamma(a))}^{\sigma} a$.

The Addition of defense branch property states that adding a defense branch to an attacked argument should increase its rank.
Property 11 (Addition of defense branch). We say that $\sigma$ satisfies Addition of defense branch iff for any $\mathcal{A} \mathcal{S}^{\prime}, \mathcal{A S}=$ $(\mathcal{A}, \mathcal{R}) \in \mathbf{A S}$ and any $a \in \mathcal{A}$ such that there exists an isomorphism $\gamma$ with $\mathcal{A S}^{\prime}=\gamma(\mathcal{A S})$ and $\mathcal{R}_{1}^{-}(a) \neq \emptyset$ we have $\gamma(a) \succ_{\mathcal{A S} \oplus \mathcal{A S} \mathcal{S}^{\prime} \oplus P_{+}(\gamma(a))}^{\sigma}$.

The Addition of attack branch property states that adding an attack branch to an argument should decrease its rank.

Property 12 (Addition of attack branch). We say that $\sigma$ satisfies Addition of attack branch iff for any $\mathcal{A S}, \mathcal{A S}=$ $(\mathcal{A}, \mathcal{R}) \in \mathbf{A S}$ and any $a \in \mathcal{A}$ such that there exists an isomorphism $\gamma$ with $\mathcal{A S}^{\prime}=\gamma(\mathcal{A S})$ we have $a \succ_{\mathcal{A S} \oplus \mathcal{A} \mathcal{S}^{\prime} \oplus P_{-}(\gamma(a))}^{\sigma} \gamma(a)$.

The Increase of attack branch property states that increasing the length of an attack branch of an argument $a$ should increase the ranking of $a$.

Property 13 (Increase of attack branch). We say that $\sigma$ satisfies Increase of attack branch iff for any $\mathcal{A S}, \mathcal{A S}=$ $(\mathcal{A}, \mathcal{R}) \in \mathbf{A S}$ and any $a \in \mathcal{A}$ such that there exists an isomorphism $\gamma$ with $\mathcal{A} \mathcal{S}^{\prime}=\gamma(\mathcal{A S})$, for every $S \in\left(2^{\mathcal{A}} \backslash\{\emptyset\}\right)$ such that for every $s \in S, \mathcal{R}_{1}^{-}(s)=\emptyset$, there exists a path of size $n$ from $S$ to $a$ with $n \in 2 \mathbb{N}+1$ and there is no path of size $m$ from $S$ to $a$ with $m \in 2 \mathbb{N}$ then for every $s \in S$, $\gamma(a) \succ_{\mathcal{A S} \oplus \mathcal{A} \mathcal{S}^{\prime} \oplus P_{+}(\gamma(s))}^{\sigma} a$.

The Increase of defense branch property states that increasing the length of a defense branch of an argument $a$ should decrease the ranking of $a$.

Property 14 (Increase of defense branch). We say that $\sigma$ satisfies Increase of defense branch iff for any $\mathcal{A S}^{\prime}, \mathcal{A S}=$ $(\mathcal{A}, \mathcal{R}) \in \mathbf{A S}$ and any $a \in \mathcal{A}$ such that there exists an isomorphism $\gamma$ with $\mathcal{A} \mathcal{S}^{\prime}=\gamma(\mathcal{A S})$, for every $S \in\left(2^{\mathcal{A}} \backslash\{\emptyset\}\right)$ such that for every $s \in S, \mathcal{R}_{1}^{-}(s)=\emptyset$, there exists a path of size $n$ from $S$ to $a$ with $n \in 2 \mathbb{N}$ and there is no path of size $m$ from $S$ to $a$ with $m \in 2 \mathbb{N}+1$ then for every $s \in S$, $a \succ_{\mathcal{A S} \oplus \mathcal{A} \mathcal{S}^{\prime} \oplus P_{+}(\gamma(s))}^{\sigma} \gamma(a)$.

The Attack vs full defense property states that, in an acyclic hypergraph argumentation framework, an argument with only one direct attacker should be ranked lower than an argument without any attack branch.

Property 15 (Attack vs full defense). We say that $\sigma$ satisfies Attack vs full defense iff for any $\mathcal{A S}=(\mathcal{A}, \mathcal{R}) \in$ AS that is acyclic and $a, b \in \mathcal{A}$ such that $\left|\mathcal{R}_{1}^{-}(b)\right|=$ $1, \mathcal{R}_{2}^{-}(b)=\emptyset$, there exists no $S \in\left(2^{\mathcal{A}} \backslash\{\emptyset\}\right)$ with for every $s \in S, \mathcal{R}_{1}^{-}(s)=\emptyset$ and a path of size $n$ from $S$ to $a$ with $n \in 2 \mathbb{N}+1$ then $a \succ_{\mathcal{A S}}^{\sigma} b$.

In the next properties, we make the assumption that the set of arguments should be compared w.r.t. their weakest arguments. We work under this hypothesis since, for an attack to exist, all arguments from $S$ are necessary. Of course, another aggregation function could be used - most of the corresponding definitions could easily be changed to take this change into account.

The Quality precedence property states that if an argument $a$ has an attacker $S$ such that the weakest element of $S$ is ranked higher than every weakest element of any attacker of $b$ than $a$ should be ranked lower than $b$. 
Property 16 (Quality precedence). We say that $\sigma$ satisfies Quality precedence iff for any $\mathcal{A S}=(\mathcal{A}, \mathcal{R}) \in \mathbf{A S}$ and $a, b \in \mathcal{A}$ such that there exists $S \in \mathcal{R}_{1}^{-}(a)$ and for every $S^{\prime} \in \mathcal{R}_{1}^{-}(b)$ we have $\min _{\mathcal{A S}}^{\sigma}(S) \succ_{\mathcal{A S}}^{\sigma} \min _{\mathcal{A S}}^{\sigma}\left(S^{\prime}\right)$ then $b \succ_{\mathcal{A S}}^{\sigma} a$.

Before introducing the next properties, we need to introduce a relation that compares sets of sets of arguments by inspiring ourselves from (Amgoud and Ben-Naim 2013).

Definition 8 (Group comparison). Let $\mathcal{A S}=(\mathcal{A}, \mathcal{R})$ and $G, G^{\prime} \subseteq\left(2^{\mathcal{A}} \backslash\{\emptyset\}\right)$. We say that $G \geq G^{\prime}$ iff there exists an injective function $f: G^{\prime} \rightarrow G$ such that for every $g^{\prime} \in$ $G^{\prime}, \min _{\mathcal{A S}}^{\sigma}\left(f\left(g^{\prime}\right)\right) \succeq_{\mathcal{A S}}^{\sigma} \min _{\mathcal{A S}}^{\sigma}\left(g^{\prime}\right)$.

Notation. As usual, we use the notation $G>G^{\prime}$ iff $\left|G^{\prime}\right|<$ $|G|$ or there exists $g^{\prime} \in G^{\prime}$ such that $\min _{\mathcal{A S}}^{\sigma}\left(f\left(g^{\prime}\right)\right) \succ_{\mathcal{A S}}^{\sigma}$ $\min _{\mathcal{A S}}^{\sigma}\left(g^{\prime}\right)$.

The Counter-transitivity property states that if the attackers of $a$ are better than the attackers of $b$ w.r.t. the group comparison then $b$ should be better or equal ranked than $a$.

Property 17 (Counter-transitivity). We say that $\sigma$ satisfies Counter-transitivity iff for any $\mathcal{A S}=(\mathcal{A}, \mathcal{R}) \in \mathbf{A S}$ and $a, b \in \mathcal{A}$ such that $\mathcal{R}_{1}^{-}(a) \geq \mathcal{R}_{1}^{-}(b)$ then $b \succeq_{\mathcal{A S}}^{\sigma} a$.

The Strict counter-transitivity property states that if the attackers of $a$ are better than the attackers of $b$ w.r.t. the group comparison then $b$ should be ranked strictly better than $a$.

Property 18 (Strict counter-transitivity). We say that $\sigma$ satisfies Strict counter-transitivity iff for any $\mathcal{A S}=(\mathcal{A}, \mathcal{R}) \in$ AS and $a, b \in \mathcal{A}$ such that $\mathcal{R}_{1}^{-}(a)>\mathcal{R}_{1}^{-}(b)$ then $b \succ_{\mathcal{A S}}^{\sigma} a$.

\section{The nh-categoriser}

As mentioned before, in each set of attacking arguments, all the components are necessary. Thus, an important intuition is that removing one argument from the set of attacking arguments would make the attack void. In the definition of the nh-categoriser, we thus consider the force of the set of attacking arguments to be the force of the weakest argument of the set. Of course, the approach that we use can be generalised with other aggregating methods.

Please note that we chose to generalise the h-categoriser because of several reasons: (1) the h-categoriser is a wellknown ranking-based semantics in the argumentation community, (2) it converges for every argumentation graph and (3) it satisfies a maximal (for set inclusion) number of postulates introduced by (Amgoud and Ben-Naim 2013).

Definition 9 (nh-categoriser). Let $\mathcal{A S}=(\mathcal{A}, \mathcal{R})$ be a hypergraph argumentation framework. The nh-categoriser is the function $C: \mathcal{A} \rightarrow(0,1]$ defined as, for all $a \in \mathcal{A}$ :

$$
C(a)= \begin{cases}1 & \text { if } \mathcal{R}_{1}^{-}(a)=\emptyset \\ \frac{1}{1+\sum_{S \in \mathcal{R}_{1}^{-}(a)} \min _{s \in S} C(s)} & \text { otherwise }\end{cases}
$$

The ranking on arguments obtained by the nb-categoriser is denoted by $\succeq_{\mathcal{A S}}^{n h}$, where for every $a, b \in \mathcal{A}, a \succeq_{\mathcal{A S}}^{n h} b$ iff $C(a) \geq C(b)$.
Example 3 (Example 1 Cont'd). The nh-categoriser scores of arguments are $C(a) \approx 0.38, C(b)=1, C(c)=$ $0.5, C(d) \approx 0.65$ and $C(e) \approx 0.53$. We obtain the ranking: $b \succeq_{\mathcal{A S}}^{n h} d \succeq_{\mathcal{A S}}^{n h} e \succeq_{\mathcal{A S}}^{n h} c \succeq_{\mathcal{A S}}^{n h} a$.

The ranking outputted by the nh-categoriser is expected as it only considers the minimum in each set of attacking arguments. As a result, although the argument $c$ is only attacked by the set $\{b\}$, it is ranked lower than argument $e$ which is attacked by both $\{d, c\}$ and $\{a, c\}$ because $b$ is stronger than both $c$ and $a$ combined.

In the rest of this section, we consider the argumentation framework $\mathcal{A S}=(\mathcal{A}, \mathcal{R})$ where $\mathcal{A}=\left\{a_{1}, \ldots, a_{n}\right\}$. We now answer the two following questions for the nhcategoriser: (1) "How many solutions exists?" and (2) "how to find them?"

We first transform the problem into a fixed point form. Let us consider $v \in[0,1]^{n}$ such that:

$$
v=F(v)=\left[f_{1}(v), f_{2}(v), \ldots, f_{n}(v)\right]^{T}
$$

where the function $F$ maps $[0,1]^{n}$ to $[0,1]^{n}$, and for every $i \in\{0, \ldots, n\}$, the function $f_{i}$ from $[0,1]^{n}$ to $[0,1]$ is defined by the nh-categoriser function:

$$
f_{i}(v)= \begin{cases}1 & \text { if } \mathcal{R}_{1}^{-}\left(a_{i}\right)=\emptyset \\ \frac{1}{1+\sum_{S \in \mathcal{R}_{1}^{-}\left(a_{i}\right)} \min _{a_{j} \in S} f_{j}(v)} & \text { else }\end{cases}
$$

The function $F$ is continuous and non-increasing as for every two vectors $u=\left(u_{1}, \ldots, u_{n}\right)$ and $u^{\prime}=\left(u_{1}^{\prime}, \ldots u_{n}^{\prime}\right)$ of $[0,1]^{n}$ with $u_{1} \leq u_{1}^{\prime}, \ldots, u_{n} \leq u_{n}^{\prime}, F(u) \geq F\left(u^{\prime}\right)$ holds.

Proposition 1 (Solution existence). For any argumentation framework $\mathcal{A S}=(\mathcal{A}, \mathcal{R})$, the nh-categoriser valuation defined in (2) has at least one solution in $[0,1]^{n}$.

Proof. The proof is similar to the one of $\mathrm{Pu}$ et al. (2014) and relies on the equivalence result that function $F$ has at least one fixed point. The proof uses Brouwer's fixed point theorem and the fact that $[0,1]^{n}$ is homeomorphic to a closed ball and function that $F$ is continuous on it.

Proposition 2 (Uniqueness of nh-categoriser valuation). Let $\mathcal{A S}=(\mathcal{A}, \mathcal{R})$ be a hypergraph argumentation framework with $\mathcal{A}=\left\{a_{1}, \ldots, a_{n}\right\}$ and $\mathcal{R}$ with sets of attacking arguments. Then, the scores of the nh-categoriser converge toward a unique solution $v^{*} \in[0,1]^{n}$, which is the limit of the sequence of $\left\{v^{(k)}\right\}_{k=0}^{\infty}$ defined from an arbitrary selected $v^{(0)}$ in $[0,1]^{n}$ and, for each $k \geq 1$, generated by

$$
v^{(k)}=F\left(v^{(k-1)}\right)
$$

Proof. First, let us consider that $u^{(0)}=(0, \ldots, 0), u^{(1)}=$ $F\left(u^{(0)}\right)=(1, \ldots, 1)$ and $u^{(k)}=F\left(u^{(k-1)}\right)$ for each $k \geq 2$. We can easily check that

$$
u^{(0)} \leq u^{(2)} \leq u^{(1)}
$$

and that there exists $0<\varphi<1$ such that

$$
\varphi u^{(1)} \leq u^{(2)}
$$


This is true because every element of $u^{(2)}$ is strictly positive and $\varphi$ can be initialised to the minimum element.

Now, let us prove by induction that for all $k \geq 0$, the following statement holds

$$
\begin{aligned}
u^{(0)} \leq u^{(2)} \leq \cdots \leq u^{(2 k)} \leq & \cdots \leq u^{(2 k+1)} \\
& \leq \cdots \leq u^{(3)} \leq u^{(1)}
\end{aligned}
$$

Base case: We showed in (4) that $u^{(0)} \leq u^{(2)} \leq u^{(1)}$.

Inductive step: Suppose that $u^{(0)} \leq u^{(2)} \leq \cdots \leq$ $u^{(2 k)} \leq \cdots \leq u^{(2 k+1)} \leq \cdots \leq u^{(3)} \leq u^{(1)}$ is true. We need to prove that $u^{(2 k)} \leq u^{(2 k+\overline{2})} \leq u^{(\overline{2 k}+3)} \leq u^{(2 k+1)}$.

First, we show that $u^{(2 k+2)} \leq u^{(2 k+1)}$. Since $u^{(2 k)} \leq$ $u^{(2 k+1)}$ and that $F$ is non increasing, we deduce that $F\left(u^{(2 k+1)}\right) \leq F\left(u^{(2 k)}\right)$ and that $u^{(2 k+2)} \leq u^{(2 k+1)}$. Likewise, we show $u^{(2 k)} \leq u^{(2 k+2)}$ using the same reasoning.

Second, we show that $u^{(2 k+2)} \leq u^{(2 k+3)} \leq u^{(2 k+1)}$. Since we prove in the previous step that $u^{(2 k+2)} \leq u^{(2 k+1)}$ and $u^{(2 k)} \leq u^{(2 k+2)}$, we deduce using the fact that $F$ is non increasing that $u^{(2 k+2)} \leq u^{(2 k+3)}$ and $u^{(2 k+3)} \leq u^{(2 k+1)}$ respectively. This concludes the proof by induction.

From (5) and (6), we find that there exists $\varphi$ such that $\varphi u^{(2 k-1)} \leq u^{(2 k)}$ for each $k \geq 1$. Now, let us denote $\pi_{k}=$ $\sup \left\{\pi\right.$ such that $\left.\pi u^{(2 k-1)} \leq u^{2 k)}\right\}$. Then, $0<\varphi \leq \pi_{1} \leq$ $\cdots \leq \pi_{k} \leq \cdots \leq 1$. We now show that $\lim _{k \rightarrow \infty} \pi_{k}=1$.

We first show $f_{i}(\pi u)=\frac{1}{\pi+f_{i}(u)(1-\pi)} f_{i}(u)$ for all $i \in$ $\{1,2, \ldots, n\}$.

$$
\begin{aligned}
& f_{i}(\pi u)=\frac{1}{1+\sum_{S \in \mathcal{R}_{1}^{-}\left(a_{i}\right)} \min _{a_{j} \in S} f_{j}(\pi u)} \\
& =\frac{1}{1+\pi \sum_{S \in \mathcal{R}_{1}^{-}\left(a_{i}\right)} \min _{a_{j} \in S} f_{j}(u)} \\
& =\frac{1+\sum_{S \in \mathcal{R}_{1}^{-}\left(a_{i}\right)} \min _{a_{j} \in S} f_{j}(u)}{1+\pi \sum_{S \in \mathcal{R}_{1}^{-}\left(a_{i}\right)} \min _{a_{j} \in S} f_{j}(u)} \times f_{i}(u) \\
& =\frac{1}{1+\pi \sum_{S \in \mathcal{R}_{1}^{-}\left(a_{i}\right)} \min _{a_{j} \in S} f_{j}(u)} \times f_{i}(u) \\
& =\frac{1}{\left(1+\sum_{S \in \mathcal{P}^{-}} \min _{a_{j} \in S} f_{j}(u)\right) \pi-\pi+1} \times f_{i}(u) \\
& \frac{S \in \mathcal{R}_{1}^{-}\left(a_{i}\right)}{1+\sum_{S \in \mathcal{R}_{1}^{-}\left(a_{i}\right)} \min _{a_{j} \in S} f_{j}(u)} \\
& =\frac{1}{\pi+\frac{1-\pi}{1+\sum_{S \in \mathcal{R}_{1}^{-}\left(a_{i}\right)} \min _{a_{j} \in S} f_{j}(u)}} \times f_{i}(u) \\
& =\frac{1}{\pi+(1-\pi) f_{i}(u)} \times f_{i}(u)
\end{aligned}
$$

Then, there exists $0<\alpha<1$ and a continuous function $\psi(\pi)=\frac{1}{\pi+\alpha(1-\pi)}$ such that

$$
F(\pi u) \leq \psi(\pi) F(u), \forall \pi \in\left[\varphi, 1\left[, u \in[\varphi, 1]^{n}\right.\right.
$$

Then, we show that

$$
\begin{array}{r}
u^{(2 k+1)}=F\left(u^{(2 k)}\right) \leq F\left(\pi_{k} u^{(2 k-1)}\right) \leq \psi\left(\pi_{k}\right) u^{(2 k)} \\
\leq \psi\left(\pi_{k}\right) u^{(2 k+2)}
\end{array}
$$

By definition of $\pi_{k}$, it holds that $\pi_{k} u^{(2 k-1)} \leq$ $u^{(2 k)}$ and since $F$ is non decreasing, we deduce that $F\left(u^{(2 k)}\right) \leq F\left(\pi_{k} u^{(2 k-1)}\right)$. Using (7), we conclude that $F\left(\pi_{k} u^{(2 k-1)}\right) \leq \pi_{k} F\left(u^{(2 k-1)}\right)=\pi_{k} u^{(2 k)}$. Using (6), we have that $u^{(2 k)} \leq u^{(2 k+2)}$ and $\pi_{k} u^{(2 k)} \leq \pi_{k} u^{(2 k+2)}$.

Now, we show that $\pi_{k+1} \geq \frac{1}{\psi\left(\pi_{k}\right)}$ by contradiction. Suppose that this $\pi_{k+1}<\frac{1}{\psi\left(\pi_{k}\right)}$. Using (8), we have that $u^{(2 k+1)} \leq \psi\left(\pi_{k}\right) u^{(2 k+2)}$ and thus $\frac{1}{\psi\left(\pi_{k}\right)} u^{(2 k+1)} \leq u^{(2 k+2)}$. Contradiction with the definition of $\pi_{k+1}$.

We now show that

$$
\begin{aligned}
1-\pi_{k+1} & \leq(1-\alpha)\left(1-\pi_{k}\right) \leq \ldots \\
& \leq(1-\alpha)^{k}\left(1-\pi_{1}\right) \leq(1-\alpha)^{k}(1-\varphi)
\end{aligned}
$$

Using the previous result, we have that

$$
\begin{aligned}
\pi_{k+1} & \geq \frac{1}{\psi\left(\pi_{k}\right)}=\pi_{k}+\alpha\left(1-\pi_{k}\right) \\
\alpha \pi_{k}-\alpha-\pi_{k}+1 & \geq 1-\pi_{k+1} \\
(1-\alpha)\left(1-\pi_{k}\right) & \geq 1-\pi_{k+1}
\end{aligned}
$$

This is sufficient to prove (9) by noticing that $\varphi \leq \pi_{1}$. As $0<\alpha<1$, thus by (9) we have

$$
\lim _{k \rightarrow \infty}\left(1-\pi_{k+1}\right)=0 \Rightarrow \lim _{k \rightarrow \infty} \pi_{k}=1
$$

There by (6), we get for any integer $p \geq 1$

$$
\begin{aligned}
0 \leq u^{(2 k+2 p)}- & u^{(2 k)} \leq u^{(2 k+1)}-u^{(2 k)} \\
& \leq\left(1-\pi_{k}\right) u^{(2 k+1)} \leq\left(1-\pi_{k}\right) u^{(1)}
\end{aligned}
$$

Since $[0,1]^{n}$ is normal, both $\left\{u^{(2 k+1)}\right\}_{k=0}^{\infty}$ and $\left\{u^{(2 k)}\right\}_{k=1}^{\infty}$ are convergence sequences. By (10) and (11), thus, there exists $u^{*} \in[0,1]^{n}$ such that

$$
\lim _{k \rightarrow \infty} u^{(2 k+1)}=\lim _{k \rightarrow \infty} u^{(2 k)}=u^{*}
$$

Hence, $u^{(2 k)} \leq u^{*} \leq u^{(2 k+1)}$ and $u^{(2 k)} \leq F\left(u^{*}\right) \leq$ $u^{(2 k+1)}$. Letting $\bar{k} \rightarrow \infty$ and combining with (12), it follows that $F\left(u^{*}\right)=u^{*}$ (it is a fixed point of $F$.

We now show that the result holds for any arbitrary $v^{(0)} \in$ $[0,1]^{n}$. By induction, we have that for any $k \geq 1, u^{(2 k)} \leq$ $v^{(2 k)} \leq u^{(2 k-1)}$ and $u^{(2 k)} \leq v^{(2 k+1)} \leq u^{(2 k+1)}$. Then 


\begin{tabular}{|c|c|}
\hline Properties & nh-categoriser \\
\hline Abstraction & $\checkmark$ \\
Independence & $\checkmark$ \\
Void precedence & $\checkmark$ \\
Self-contradiction & $x$ \\
Cardinality precedence & $x$ \\
Defense precedence & $\checkmark$ \\
Total & $\checkmark$ \\
Non-attacked equiv. & $\checkmark$ \\
Distributed-defense precedence & $x$ \\
Strict addition of defense branch & $x$ \\
Addition of defense branch & $x$ \\
Addition of attack branch & $\checkmark$ \\
Increase of attack branch & $\checkmark$ \\
Increase of defense branch & $\checkmark$ \\
Attack vs full defense & $\boldsymbol{\checkmark}$ \\
Quality precedence & $\boldsymbol{x}$ \\
Counter-transitivity & $\checkmark$ \\
Strict counter transitivity & $\checkmark$ \\
\hline
\end{tabular}

Table 1: Properties satisfied by the nh-categoriser

$v^{(k)} \rightarrow v^{*}=u^{*}$ as $k \rightarrow \infty$. In particular, let $v^{(0)}=w^{*}$, where $w^{*}$ is any fixed point of $F$ in $[0,1]^{n}$, then $v^{(k)}=w^{*}$ for all $k \geq 1$, and we get $w^{*}=u^{*}$. So $F$ has a unique fixed point in $[0,1]^{n}$.

In Table 1, we show that nh-categoriser satisfies the same properties as h-categoriser. The proof is dropped because of lack of space.

Our goal is achieved as the nh-categoriser is a proper instantiation of the h-categoriser for hypergraph argumentation frameworks and satisfies the same properties as the regular h-categoriser. The set of properties of this paper can be used to select the most suitable ranking-based semantics for a specific task. There has been a lot of discussion about whether some properties are more desirable than others, for instance, the satisfaction of Abstraction is considered essential for most ranking-based semantics whereas other properties, such as Increase of attack branch, are considered optional.

In the next proposition, we show that there always exists a regular Dung's abstract argumentation framework such that the score on arguments with the h-categoriser are the same as the scores on arguments in the hypergraph argumentation framework with the nh-categoriser.

Definition 10 (Abstract argumentation framework). An abstract argumentation framework is a pair $\mathcal{A S}^{\star}=\left(\mathcal{A}^{\star}, \mathcal{R}^{\star}\right)$, where $\mathcal{A}^{\star}$ is a finite and non-empty set of arguments and $\mathcal{R}^{\star} \subseteq \mathcal{A} \times \mathcal{A}$ is a set of binary attacks.

Notation. Let $\mathcal{A S}^{\star}=\left(\mathcal{A}^{\star}, \mathcal{R}^{\star}\right)$ be an abstract argumentation framework and $a \in \mathcal{A}^{\star}, \operatorname{Att}(a)=\left\{b \in \mathcal{A}^{\star} \mid(b, a) \in\right.$ $\left.\mathcal{R}^{\star}\right\}$.

Definition 11 (h-categoriser). Let $\mathcal{A} \mathcal{S}^{\star}=\left(\mathcal{A}^{\star}, \mathcal{R}^{\star}\right)$ be an abstract argumentation framework. The h-categoriser is the function $C^{\prime}: \mathcal{A}^{\star} \rightarrow[0,1]$ defined as, for all $a \in \mathcal{A}^{\star}$ :

$$
C^{\prime}(a)= \begin{cases}1 & \text { if } \operatorname{Att}(a)=\emptyset \\ \frac{1}{1+\sum_{b \in \operatorname{Att}(a)} C^{\prime}(b)} & \text { otherwise }\end{cases}
$$

Proposition 3 (Dung Equivalent Existence). Let $\mathcal{A S}=$ $(\mathcal{A}, \mathcal{R})$ be a hypergraph argumentation frameworks. Then, there exists at least one abstract argumentation framework $\mathcal{A S}^{\star}=\left(\mathcal{A}^{\star}, \mathcal{R}^{\star}\right)$, a subset $\left\{a_{1}, \ldots, a_{m}\right\} \subseteq \mathcal{A}^{\star}$ and a bijection $\gamma: \mathcal{A} \rightarrow\left\{a_{1}, \ldots, a_{m}\right\}$ such that for every $a \in \mathcal{A}$, $C(a)=C^{\prime}(\gamma(a))$.

Proof. This proof is an original work and is split in two parts: First, we exhibit how to build an abstract argumentation framework $\mathcal{A S}^{\star}$ from an arbitrary hypergraph argumentation framework $\mathcal{A S}$. Second, we show that aforementioned $\mathcal{A S}^{\star}$ is such that Proposition 3 is satisfied.

- 1- Let $\mathcal{A S}=(\mathcal{A}, \mathcal{R})$ be an arbitrary hypergraph argumentation framework. We build the abstract argumentation $\mathcal{A S}^{\star}=\left(\mathcal{A}^{\star}, \mathcal{R}^{\star}\right)$ such that:

- $\mathcal{A}^{\star}=\left\{a_{1}, \ldots, a_{m}\right\} \cup\left\{a_{m+1}, \ldots, a_{n}\right\}$

- There is a bijection $\gamma_{1}$ from $\mathcal{A}$ to $\left\{a_{1}, \ldots, a_{m}\right\}$ and a bijection $\gamma_{2}$ from $\mathcal{R}$ to $\left\{a_{m+1}, \ldots, a_{n}\right\}$.

- For every $(S, t) \in \mathcal{R}$, we create the attack $\left(\gamma_{2}((S, t)), \gamma_{1}(t)\right)$ in $\mathcal{R}^{\star}$.

- For every $(S, t) \in \mathcal{R}$, we consider the set $X_{S}=\{s \in$ $S \mid$ for every $\left.s^{\prime} \in S, s^{\prime} \succeq_{\mathcal{A S}}^{n h} s\right\}$ and we chose $x_{S} \in$ $X_{S}$. Then, for every $S^{\prime} \in \mathcal{R}_{1}^{-}\left(x_{S}\right)$, we create the attack $\left(\gamma_{2}\left(\left(S^{\prime}, x_{S}\right)\right), \gamma_{2}((S, t))\right)$ in $\mathcal{R}^{\star}$.

- 2- We now show that the constructed argumentation framework $\mathcal{A S}^{\star}$ is such that Proposition 3 is satisfied. Let $X^{\prime}=\left\{a_{m+1}, \ldots, a_{n}\right\}$ and $\mathcal{A S}^{\prime}=\left(\mathcal{A}^{\prime}, \mathcal{R}^{\prime}\right)$ be the restriction of $\mathcal{A S}^{\star}$ to $X^{\prime}$, i.e. $\mathcal{A}^{\prime}=X^{\prime}$ and $\mathcal{R}^{\prime}=\{(a, b) \in$ $\mathcal{R}^{\star} \mid a \in X^{\prime}$ and $\left.b \in X^{\prime}\right\}$.

We now define the function $f: X^{\prime} \rightarrow \mathbb{R}$ that returns for every element $a$ in $X^{\prime}$, the minimum score of the attackers associated with the attack corresponding to $a$. Namely, for every $a \in X^{\prime}, f(a)=\min _{a^{\prime} \in W} C\left(a^{\prime}\right)$ where $(W, t)=\gamma_{2}^{-1}(a)$ and $\gamma_{2}^{-1}$ is the inverse function of $\gamma_{2}$. We now prove that for every $a \in \mathcal{A}^{\prime}$, it holds that $f(a)=\frac{1}{1+\sum_{(b, a) \in \mathcal{R}^{\prime}} f(b)}$. Let $a$ be an arbitrary argument in $\mathcal{A}^{\prime},\left\{b_{1}, \ldots, b_{p}\right\}$ the set of attackers of $a$ in $\mathcal{A S}^{\prime}$ and $(W, t)=\gamma_{2}^{-1}(a)$. By construction, we know that there exists $x_{W} \in W$ such that $\left\{b_{1}, \ldots, b_{p}\right\}=$ $\left\{\gamma_{2}\left(\left(S^{\prime}, x_{W}\right)\right) \mid S^{\prime} \in \mathcal{R}_{1}^{-}\left(x_{W}\right)\right\}$. Thus,

$$
\begin{aligned}
f(a) & =\min _{a^{\prime} \in W} C\left(a^{\prime}\right) \\
f(a) & =C\left(x_{W}\right) \\
f(a) & =\frac{1}{1+\sum_{S^{\prime} \in R_{1}^{-}\left(x_{W}\right)} \min _{s^{\prime} \in S^{\prime}} C\left(s^{\prime}\right)} \\
f(a) & =\frac{1}{1+\sum_{(b, a) \in \mathcal{R}^{\prime}} f(b)}
\end{aligned}
$$


Since $f$ satisfies the same formula as h-categoriser then $f$ is identical to h-categoriser on $\mathcal{A S}^{\prime}$ (Pu et al. 2014).

Let us now consider the whole argumentation graph $\mathcal{A S}^{\star}$. Since there are no attacks from arguments in $\left\{a_{1}, \ldots, a_{m}\right\}$ to arguments in $\left\{a_{m+1}, \ldots, a_{n}\right\}$, the $\mathrm{h}$ categoriser gives the same scores to $\left\{a_{m+1}, \ldots, a_{n}\right\}$ in $\mathcal{A S}^{\star}$ and in $\mathcal{A S}^{\prime}$. Since for every argument $a$ in $\left\{a_{1}, \ldots, a_{m}\right\}$, the set of attackers of $a$ in $\mathcal{A S}^{\star}$ is $\{b \in$ $\left\{a_{m+1}, \ldots, a_{n}\right\} \mid \gamma_{2}^{-1}(b)=\left(S, \gamma_{1}^{-1}(a)\right)$ with $S \in$ $\left.\mathcal{R}_{1}^{-}\left(\gamma_{1}^{-1}(a)\right)\right\}$, we have for every $a \in\left\{a_{1}, \ldots, a_{m}\right\}$ :

$$
\begin{aligned}
& C^{\prime}(a)=\frac{1}{1+\sum_{b \in \operatorname{Att}(a)} C^{\prime}(b)} \\
& C^{\prime}(a)=\frac{1}{1+\sum_{b \in \operatorname{Att}(a)} f(b)} \\
& C^{\prime}(a)=\frac{1}{1+\sum_{b \in \operatorname{Att}(a)} \min _{a^{\prime} \in W} C\left(a^{\prime}\right)} \text { where }(W, t)=\gamma_{2}^{-1}(b) \\
& C^{\prime}(a)=\frac{1}{1+\sum_{S \in \mathcal{R}_{1}^{-}\left(\gamma_{1}^{-1}(a)\right)} \min _{s \in S} C(s)} \\
& C^{\prime}(a)=C\left(\gamma_{1}^{-1}(a)\right)
\end{aligned}
$$

Proposition 3 is important as it indicates the computational relation between the nh-categoriser and the $\mathrm{h}$ categoriser. Moreover, this proposition shows how one is able to move from an argumentation hypergraph to a regular argumentation directed graph without loss of generality.

Example 4 (Example 3 Cont'd). Let us consider the abstract argumentation framework $\mathcal{A S}^{\star}=\left(\mathcal{A}^{\star}, \mathcal{R}^{\star}\right)$ with $\mathcal{A}^{\star}=$ $\left\{a^{\prime}, b^{\prime}, c^{\prime}, d^{\prime}, e^{\prime}\right\} \cup\left\{d_{a}, b_{a}, a c_{e}, b_{c}, b e_{d}, d c_{e}\right\}$ and $\mathcal{R}^{\star}=$ $\left\{\left(d_{a}, a\right),\left(d_{a}, a c_{e}\right),\left(b_{a}, a\right),\left(b_{a}, a c_{e}\right),\left(b_{c}, c\right),\left(b_{c}, d c_{e}\right),\left(b e_{d}\right.\right.$, d), $\left.\left(b e_{d}, d_{a}\right),\left(d c_{e}, e\right),\left(d c_{e}, b e_{d}\right),\left(a c_{e}, e\right),\left(a c_{e}, b e_{d}\right)\right\}$. If $\gamma$ is the bijection from $\mathcal{A}$ to $\mathcal{A}^{\star}$ with $\gamma(a)=a^{\prime}, \gamma(b)=$ $b^{\prime}, \gamma(c)=c^{\prime}, \gamma(d)=d^{\prime}$ and $\gamma(e)=e^{\prime}$ then it holds that for every $a \in \mathcal{A}, C(a)=C^{\prime}(\gamma(a))$.

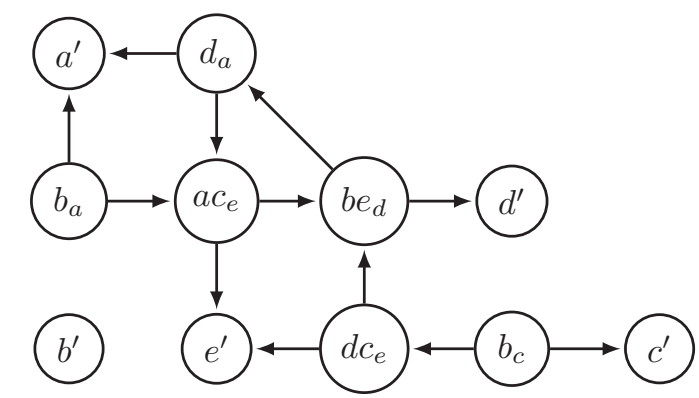

Figure 2: An abstract argumentation framework

We would like to point out that for a hypergraph argumentation framework, there might be several abstract argumentation frameworks that satisfy Proposition 3.
Please note that although it seems intuitive that restricting the sets of attacking arguments to a binary attack from the weaker argument of the set would result in the same score with the h-categoriser than with the nh-categoriser, this idea does not work. For illustration purposes, we present one simple counter-example against this intuition. Consider the argumentation framework $\mathcal{A S}=(\mathcal{A}, \mathcal{R})$ with such that $\mathcal{A}=\{a, b, c, d\}$ and $\mathcal{R}=\{(\{d\}, b),(\{b, a\}, c),(\{d, b\}, c)\}$. The score of the argument $c$ in $\mathcal{A S}$ using the nh-categoriser is $C(c)=0.5$. Now, if we use the aforementioned approach, we obtain the binary argumentation framework $\mathcal{A S}^{\star}=$ $\left(\mathcal{A}, \mathcal{R}^{\star}\right)$ such that $\mathcal{R}^{\star}=\{(b, c),(d, b)\}$. In $\mathcal{A S}^{\star}$, the score of $c$ with the h-categoriser is $C^{\prime}(c)=0.66$, which is not equal to the score of $c$ given by the nh-categoriser. The approach described in the proof of Proposition 3 enables us to obtain a strength of 0.5 , which is the strength of $c$ using the nh-categoriser.

\section{Discussion and Related Work}

We introduced the notion of ranking-based semantics for argumentation frameworks with sets of attacking arguments (also called hypergraph argumentation frameworks). We motivated our work rooted in the need to account for extensions of classical Dung graphs encoding arguments over a logical language not necessarily closed under the "and" logical connective. In that context, our work aligns with existing works in the literature that extend ranking-based semantics to weighted argumentation framework (Amgoud et al. 2017), support argumentation frameworks (Amgoud and Ben-Naim 2016) or bipolar argumentation frameworks (Amgoud et al. 2008). Our contributions consist in (1) providing a set of 18 translated properties for ranking-based semantics that are directly applicable in the context of hypergraph argumentation framework, (2) introducing the nhcategoriser as the first ranking-based semantics for hypergraph argumentation framework, (3) giving the complete proof of the existence of a unique solution for the nhcategoriser and (4) bridging the link between ranking arguments in hypergraph argumentation frameworks and ranking arguments in the usual Dung's framework.

The focus of the argumentation community have recently been shifted to argumentation frameworks with sets of attacking arguments (Flouris and Bikakis 2019) and we believe that this paper will be of interest for the community.

Although the work of (Flouris and Bikakis 2019) completes the characterisation of such argumentation frameworks with the treatment of various interesting semantics not considered in the original publication (Nielsen and Parsons 2007) and functions allowing the transition between extensions and labellings along with their properties, it does not consider ranking-based semantics. To the extend of our knowledge, this paper is the only work that has been interested in the topic of applying ranking-based semantics to this setting.

\section{Acknowledgments}

We acknowledge the support of the DOCAMEX project. 


\section{References}

Amgoud, L., and Ben-Naim, J. 2013. Ranking-Based Semantics for Argumentation Frameworks. In Scalable Uncertainty Management - 7th International Conference, SUM 2013, Washington, DC, USA, September 16-18, 2013. Proceedings, 134-147.

Amgoud, L., and Ben-Naim, J. 2016. Evaluation of Arguments from Support Relations: Axioms and Semantics. In Proceedings of the Twenty-Fifth International Joint Conference on Artificial Intelligence, IJCAI 2016, New York, NY, USA, 9-15 July 2016, 900-906.

Amgoud, L.; Cayrol, C.; Lagasquie-Schiex, M.-C.; and Livet, P. 2008. On bipolarity in argumentation frameworks. Int. J. Intell. Syst. 23(10):1062-1093.

Amgoud, L.; Ben-Naim, J.; Doder, D.; and Vesic, S. 2017. Acceptability Semantics for Weighted Argumentation Frameworks. In Proceedings of the Twenty-Sixth International Joint Conference on Artificial Intelligence, IJCAI 2017, Melbourne, Australia, August 19-25, 2017, 56-62.

Baroni, P.; Caminada, M.; and Giacomin, M. 2011. An introduction to argumentation semantics. Knowledge Eng. Review 26(4):365-410.

Besnard, P., and Hunter, A. 2001. A logic-based theory of deductive arguments. Artif. Intell. 128(1-2):203-235.

Bonzon, E.; Delobelle, J.; Konieczny, S.; and Maudet, N. 2016. A Comparative Study of Ranking-Based Semantics for Abstract Argumentation. In Proceedings of the Thirtieth AAAI Conference on Artificial Intelligence, February 12-17, 2016, Phoenix, Arizona, USA., 914-920.

Cayrol, C., and Lagasquie-Schiex, M.-C. 2005. Graduality in Argumentation. J. Artif. Intell. Res. (JAIR) 23:245-297.

Dung, P. M. 1995. On the Acceptability of Arguments and its Fundamental Role in Nonmonotonic Reasoning, Logic Programming and n-Person Games. Artif. Intell. 77(2):321358.

Flouris, G., and Bikakis, A. 2019. A comprehensive study of argumentation frameworks with sets of attacking arguments. International Journal of Approximate Reasoning 109:5586.

Leite, J., and Martins, J. 2011. Social Abstract Argumentation. In IJCAI 2011, Proceedings of the 22nd International Joint Conference on Artificial Intelligence, Barcelona, Catalonia, Spain, July 16-22, 2011, 2287-2292.

Matt, P.-A., and Toni, F. 2008. A Game-Theoretic Measure of Argument Strength for Abstract Argumentation. In Logics in Artificial Intelligence, 11th European Conference, JELIA 2008, Dresden, Germany, September 28 - October 1, 2008. Proceedings, 285-297.

Nielsen, S. H., and Parsons, S. 2007. A Generalization of Dung's Abstract Framework for Argumentation: Arguing with Sets of Attacking Arguments. In Maudet, N.; Parsons, S.; and Rahwan, I., eds., Argumentation in Multi-Agent Systems, 54-73. Springer Berlin Heidelberg.

Pu, F.; Luo, J.; Zhang, Y.; and Luo, G. 2014. Argument Ranking with Categoriser Function. CoRR abs/1406.3877.
Verheij, B. 1996. Rules, Reasons, Arguments. Formal studies of argumentation and defeat. Ph.D. Dissertation, University of Maastricht. 